\title{
Article
}

\section{Bell Violation in Primordial Cosmology}

\author{
Sayantan Choudhury ${ }^{1, *}$, Sudhakar Panda ${ }^{2,3}$ and Rajeev Singh ${ }^{4}$ \\ 1 Department of Theoretical Physics, Tata Institute of Fundamental Research, Colaba, Mumbai 400005, India \\ Institute of Physics, Sachivalaya Marg, Bhubaneswar, Odisha 751005, India; panda@iopb.res.in \\ Homi Bhabha National Institute, Training School Complex, Anushakti Nagar, Mumbai 400085, India \\ 4 Department of Physics, Savitribai Phule Pune University, Pune 411007, India; rajeevsingh240291@gmail.com \\ * Correspondence: sayantan@theory.tifr.res.in or sayanphysicsisi@gmail.com; Tel.: +91-22-2278-2223
}

Academic Editors: Mariusz P. Dąbrowski, Manuel Krämer and Vincenzo Salzano

Received: 27 December 2016; Accepted: 8 February 2017; Published: 17 February 2017

\begin{abstract}
In this paper, we have worked on the possibility of setting up an Bell's inequality violating experiment in the context of primordial cosmology following the fundamental principles of quantum mechanics. To set up this proposal, we have introduced a model-independent theoretical framework using which we have studied the creation of new massive particles for the scalar fluctuations in the presence of an additional time-dependent mass parameter. Next we explicitly computed the one-point and two-point correlation functions from this setup. Then, we comment on the measurement techniques of isospin breaking interactions of newly introduced massive particles and its further prospects. After that, we give an example of the string theory-originated axion monodromy model in this context. Finally, we provide a bound on the heavy particle mass parameter for any arbitrary spin field.
\end{abstract}

Keywords: de-Sitter vacua; string theory; string cosmology; axion; quantum information

\section{Introduction}

According to the inflationary model, the primordial fluctuations which we see in $\mathrm{CMB}$ (Cosmic Microwave Background) were produced by quantum mechanical effects in the early universe. These fluctuations are the origin for the formation of large-scale structure, but the fluctuations we observe at present are actually classical in nature. The highly-entangled quantum mechanical wave function of the universe plays a very important role during quantum mechanical interpretation of the required fluctuations. Hence, one can use the Hartle-Hawking wave function in de-Sitter space. Due to this fact, quantum mechanical fluctuations can be theoretically demonstrated, and can also be implemented in the context of primordial cosmology -if and only if we perform a cosmological experiment using the highly-entangled quantum mechanical wave function of the universe which is defined in inflationary period and eventually violate Bell's inequality [1]. To describe the background methodology, it is important to mention that in the context of quantum mechanics, Bell test experiment can be described by the measurement of two non- commutating physical operators. These operators are associated with two distinct locations in space-time. Thus, using same analogy in the context of primordial cosmology, we can perform cosmological observations on two spatially separated and causally disconnected places up to the epoch of reheating (after inflation). During these observations we can measure the numerical values of various cosmological observables (along with cosmic variance), and can also be computed from scalar curvature fluctuation. However, it is important to note that for all such observations, we cannot measure the value of associated canonically conjugate momentum. Hence, for these cosmological observables, we cannot measure the imprints of two non-commuting operators in primordial cosmology. There is subtle point, however, which is that if these observables satisfy the minimum requirements of decoherence effect, then we can possibly perform measurements 
from two exactly commuting cosmological observables, and therefore we will be able to design a Bell's inequality violating cosmological experimental setup. We know that in quantum theory, to design such an experimental setup one has to perform a number of repeated measurements on the same object (which in this context is the same quantum state of the universe), and therefore in such a physical situation we can justify the appearance of each and every measurement using a single quantum state. In the case of primordial cosmology, we can do the same thing; that is, consider two spatially-separated portions in the sky which plays the same role of performing repeated cosmological Bell's inequality violating experiment using the same quantum mechanical state. Therefore, we have the advantage of choosing the required properties of two spatially-separated portions in the sky in order to setup Bell's inequality violating experimental setup. It is completely possible to set up a Bell's inequality violating cosmological experimental setup if we can find a link which connects these non-commutating cosmological observables and classical probability distribution function originated from model of inflation (see Reference [2-4] for more details). In this article, we explore this possibility in detail. See also References [5-8], where the authors have also studied various consequences of the violation of Bell's inequalities in other areas.

\section{Bell's Inequality and its Violation in Quantum Mechanics}

John Stewart Bell in Reference [1] showed that "In a theory in which parameters are added to quantum mechanics to determine the results of individual measurements, without changing the statistical predictions, there must be a mechanism whereby the setting of one measuring device can influence the reading of another instrument, however remote". In that paper, J.S Bell gave two assumptions for his theory, which were (1) concept of reality; and (2) concept of locality. Using these assumptions, he derived an inequality, which is known as "Bell's Inequality". In this paper, he proved that "no local hidden variable theory is compatible with quantum mechanics". See Figure 1 for the representative setup for the spin system. Here the operators-which are $A_{0}, B_{0}, A_{1}$, and $B_{1}$-correspond to measuring the spin, and their eigenvalues are \pm 1 , choosing the value of operators as:

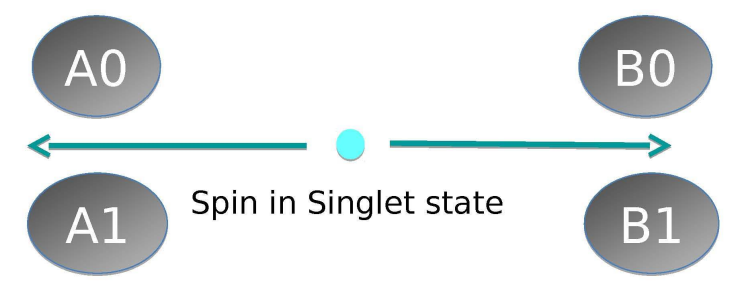

The eigen values of $\mathrm{A} 0, \mathrm{~B} 0, \mathrm{~A} 1$ and $\mathrm{B} 1$ is +1 or -1 .

Figure 1. Schematic diagram of Bell's inequality example for a spin system. Here pictures are taken from [3].

$$
A_{0}=n_{0} . \sigma, A_{1}=n_{1} . \sigma, B_{0}=n_{0} . \sigma, B_{1}=n_{1} . \sigma .
$$

Further considering a variable which is given by:

$$
\langle R\rangle=\left\langle A_{0} B_{0}\right\rangle+\left\langle A_{1} B_{0}\right\rangle+\left\langle A_{0} B_{1}\right\rangle-\left\langle A_{1} B_{1}\right\rangle
$$

According to hidden variable theory, $|\langle R\rangle| \leq 2$. In quantum mechanics, however, the expectation value of $R$ can be found bigger. By squaring Equation (2), we can show that $R^{2}=4+\left[A_{1}, A_{0}\right]\left[B_{1}, B_{0}\right] \Rightarrow$ $|\langle R\rangle|>2$, making $|\langle R\rangle|$ larger than 2, which violates Bell's inequality. The question now is how to draw the above conclusion, therefore choosing:

$$
A_{0}=x . \sigma, \quad A_{1}=y \cdot \sigma, \quad B_{0}=\sin \theta(x . \sigma)+\cos \theta(y \cdot \sigma), \quad B_{1}=\cos \theta(x . \sigma)-\sin \theta(y \cdot \sigma),
$$


we get the extra $\sqrt{2}$ factor for the maximal violation; i.e., $|\langle R\rangle|>2 \sqrt{2}$.

\section{Cosmological Bell Violating Setup}

To describe the setup of Bell violating experiment in the context of cosmology, we have to start with the quantum fluctuations, which can be treated as a time-dependent harmonic oscillator in Fourier space, given by:

$$
S=\int \frac{d \eta}{\eta^{2}}\left(\left|\partial_{\eta} \phi_{\mathbf{k}}(\eta)\right|^{2}-k^{2}\left|\phi_{\mathbf{k}}(\eta)\right|^{2}\right) .
$$

Therefore, to describe such quantum fluctuations in expanding space-time, we have to use the quasi de Sitter metric, $d s^{2}=a^{2}(\eta)\left(-d \eta^{2}+d \mathbf{x}^{2}\right)$, where $H$ is the Hubble parameter, $\mathbf{x}$ represents comoving spatial coordinates, $\eta$ signifies the conformal time coordinate, and $a(\eta)=-(1+\epsilon) / H \eta$ is the scale factor, with $\epsilon=-\dot{H} / H^{2}$. Noting that, here it can be easily shown that quantum theory plays an important role in producing spatially-dependent fluctuations in terms of the scalar fields. See Figure 2, where we have shown a comparison between a successful and an unsuccessful setup for Bell's inequality violation in quantum mechanics and cosmology.

Bell case

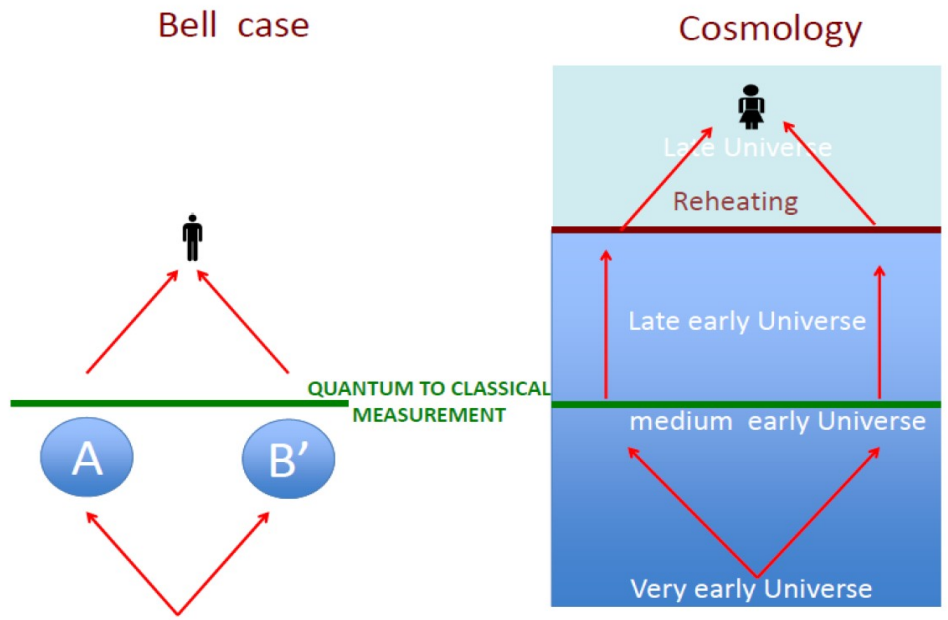

(a)
Bell case

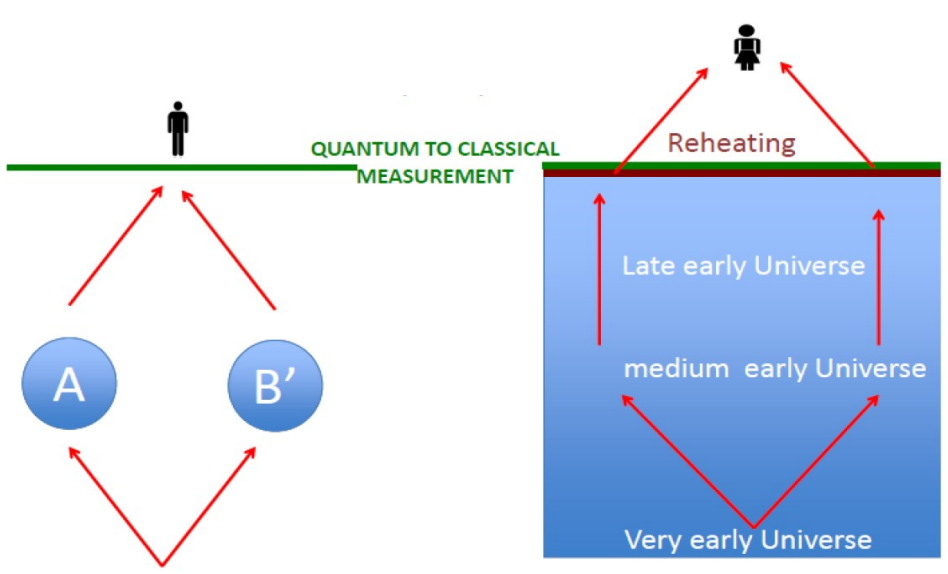

(b)

Figure 2. (a) Successful vs. (b) Unsuccessful setup for Bell's inequality violation in Quantum Mechanics and Cosmology [4]. 
We know that quantum mechanical effects are the seeds for primordial fluctuations in the early universe. However, the fluctuations we have observed today is completely classical in nature, as we said earlier. We know that in the theory of inflation, all such fluctuations become classical in nature as they exit the horizon, and inside the horizon, all of them are quantum in nature. In de Sitter space, $k^{3}\left|\left[\phi_{\mathbf{k}}(\eta), \eta \partial_{\eta} \phi_{\mathbf{k}}\right]\right| \approx H^{3}(\eta k)^{3} \rightarrow 0$ as $\eta k \rightarrow 0$ at the end of inflation, which is the signature of Bell inequality violation in our cosmological setup. Hence, when reheating occurs after inflation, a classical measure can be written down, or more precisely, a classical probability distribution function of fluctuation, as follows: $\rho[\phi(x)]=\mu[\phi(x)]=|\Psi[\phi(x)]|^{2}$. Here, $|\Psi[\phi(x)]|^{2}$, or equivalently, $\mu[\phi(x)]$ symbolizes the classical probability distribution, which is the state of the universe at the spatial hyper-surface where the process of reheating occurs. Here all the fluctuations can be treated as a distribution of classical random variables. However, from the computed classical probability distribution function $\rho[\phi(x)]$, one cannot say anything regarding the exact measurement procedure on a quantum state. This is really a disappointing fact from the theoretical point of view, but that is not the end of the proposal. Here it is important to mention that one can treat a measurement as a specific unitary evolution of the combined effect of physical system as well as measuring apparatus. Such physical states can be visualized as classical objects in the context of cosmology, and in a direct sense are not very useful (for more detail, see References [2,3]). Here we need to follow a few steps to overcome the difficulties [3].

- Choose a toy model of universe that will make the job easy.

- Here we are not claiming that this is the unique model of universe using which one can design the setup.

- Since we do not have any direct observational evidence, we also cannot claim that this toy model is our known universe. However, it may be that in the future this will be tested.

- In this toy model of the universe, we test the validity of Bell's inequality with primordial fluctuations.

- In this computation, massive particles with spin " $s$ " ( $s=0,2$, and $>2$ allowed) and additionally "isospin" quantum number plays important role.

- We provide an example for Stringy axion which has "isospin".

- Time dependent mass profile with dependence on "isospin" makes the job easy. Axions have such profile.

- Such time dependence in mass profile of the massive particles (ex. axion) produces classical perturbations on the inflaton. As a result, hot spots produced in CMB by curvature fluctuations and all such massive particles are visible today.

\section{Role of Massive New Particles}

In this section, we discuss the explicit role of massive particles in the context of Bell's inequality violation in cosmology. Here it is important to note that all the massive particles have the few characteristics to violate Bell's inequality: (A) Particle pair creation (Bell pair) is required to produce hot and cold spots in CMB; (B) Particle mass should have a time-dependent profile. Growing mass profile is usually preferred. However, sometimes other behavior is also important, depending on the specific mathematical structure of the mass profile; (C) Heavy fields become heavy at early and late times, but in an intermediate time scale where inflation occurs, it behaves like a light field; (D) Each massive particle pair is created in "isospin" singlet manner. This is required for measurement in the detectors. Time dependence of the inflation give rise to a time-dependent mass $m(\eta)$. In the present context, we consider three mass profiles as a toy model: (A) $m^{2} / H^{2}=\gamma\left(\frac{\eta}{\eta_{0}}-1\right)^{2}+\delta$; (B) $2 m^{2} / m_{0}^{2}=\left[1-\tanh \left(\frac{\rho}{H} \ln (-H \eta)\right)\right]$; and (C) $m^{2} / m_{0}^{2}=\operatorname{sech}^{2}\left(\frac{\rho}{H} \ln (-H \eta)\right)$. See Figure 3, where we have shown the behaviour of all the toy models of mass profile for massive particles with conformal time scale. The equation of motion for the massive field is [3].

$$
h_{k}^{\prime \prime}+\left\{c_{S}^{2} k^{2}+\left(\frac{m^{2}}{H^{2}}-\left[v^{2}-\frac{1}{4}\right]\right) \frac{1}{\eta^{2}}\right\} h_{k}=0,
$$


whereas, in the quasi de Sitter case, the parameter $v$ can be written as $v=\frac{3}{2}+\epsilon+\frac{\eta}{2}+\frac{s}{2}$, where $\eta$ and $s$ are defined as $\eta=\frac{\dot{e}}{H \epsilon}, s=\frac{\dot{c}_{S}}{H c_{S}}$. Here WKB approximation is approximately valid for the mode function $h_{k}$ solution. If we solve the equation of motion for the heavy fields, then we can have the solution for the fluctuations, where it is assumed that the variation in time in heavy field mass parameter is very slow. For a case which is dependent on time, it is possible depending on the mathematical structure of the heavy field mass parameter $m(\eta)$. In the standard WKB approximation, the total solution is:

$$
h_{k}(\eta)=\left[D_{1} u_{k}(\eta)+D_{2} \bar{u}_{k}(\eta)\right]
$$

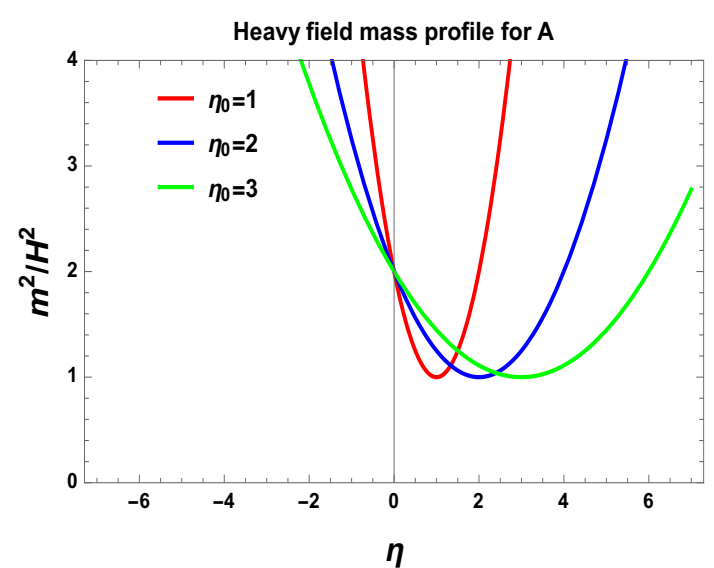

(a)

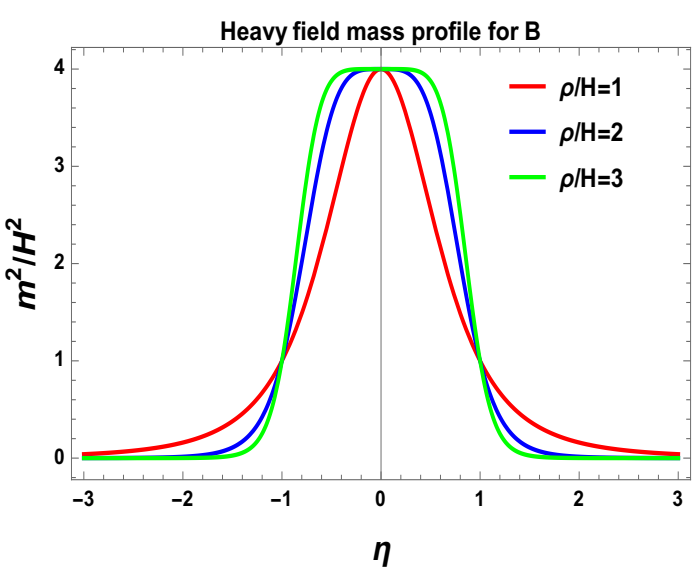

(b)

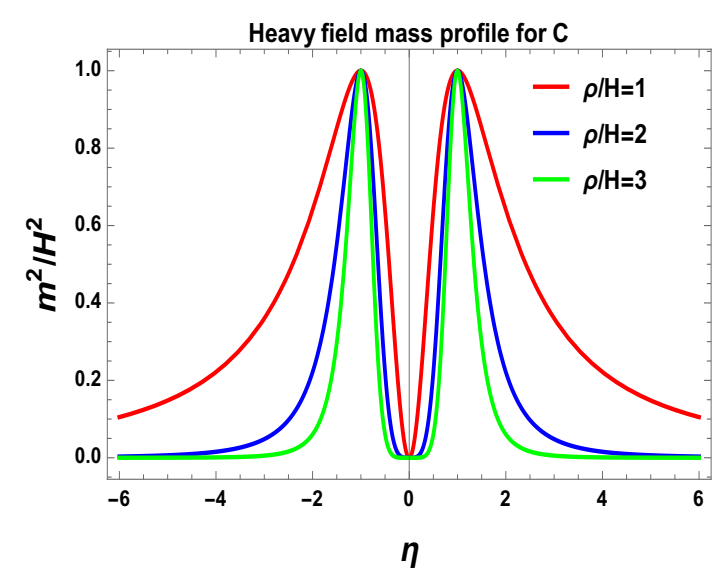

(c)

Figure 3. Toy model mass profiles for massive particles. (a) Mass profile for A with $\gamma=1$ and $\delta=1$; (b) Mass profile for B with $\frac{m_{0}^{2}}{2 H^{2}}=1$; (c) Mass profile for $\mathrm{C}$ with $\frac{m_{0}^{2}}{H^{2}}=1$. Here pictures are taken from [3].

Here, $u_{k}(\eta)$ and $\bar{u}_{k}(\eta)$ are defined as:

$$
u_{k}(\eta)=\frac{1}{\sqrt{2 p(\eta)}} \exp \left[i \int^{\eta} d \eta^{\prime} p\left(\eta^{\prime}\right)\right], \quad \bar{u}_{k}(\eta)=\frac{1}{\sqrt{2 p(\eta)}} \exp \left[-i \int^{\eta} d \eta^{\prime} p\left(\eta^{\prime}\right)\right],
$$

where total solution for $h_{k}$ is given in terms of two linearly independent solutions. Here the new conformal time-dependent factor $p(\eta)$ is defined as $p(\eta)=\sqrt{\left\{c_{S}^{2} k^{2}+\left(\frac{m^{2}}{H^{2}}-\left[v^{2}-\frac{1}{4}\right]\right) \frac{1}{\eta^{2}}\right\}}$, which we have used in our calculation, where $D_{1}$ and $D_{2}$ are two arbitrary integration constants, and these constants depend on the initial condition during WKB approximation at early and late time 
scales. In our context, these constants $D_{1}$ and $D_{2}$ can be identified with the Bogoliubov coefficient in momentum space,

$$
\begin{aligned}
& D_{1}=\beta(k)=\frac{\mathcal{R}}{\mathcal{T}}=\int_{-\infty}^{0} d \eta \frac{\left(p^{\prime}(\eta)\right)^{2}}{4 p^{3}(\eta)} \exp \left[2 i \int_{-\infty}^{\eta} d \eta^{\prime} p\left(\eta^{\prime}\right)\right], \\
& D_{2}=\alpha(k)=\frac{1}{\mathcal{T}}=\sqrt{1+|\beta(k)|^{2}} e^{i \phi},
\end{aligned}
$$

where $\mathcal{R}$ and $\mathcal{T}$ represent the reflection and transmission coefficient, respectively. Further, one can also compute the number of produced particle pairs and its energy density using the following equations:

$$
\mathcal{N}_{\text {pair }}=\frac{1}{(2 \pi a)^{3}} \int d^{3} \mathbf{k}|\beta(k)|^{2}, \quad \rho_{\text {pair }}=\frac{1}{(2 \pi a)^{3} a} \int d^{3} \mathbf{k} p(\eta)|\beta(k)|^{2} .
$$

In the present context, to describe a very small fraction of particle creation after inflation, we have to start with a Bogoliubov coefficient $\beta$ in FLRW space time, which gives the amount of mixing between the WKB approximated solutions, which is of two type. Noting that in the sub-Hubble region $\left(\left|k_{s} \eta\right|>>1\right)$ Bogoliubov coefficient $\beta$ is small, and hence the representative probability distribution $P(x)$ for the relative comoving distance $x$ between the two pairs peaks at the comoving length scale, which is $x \sim\left|\eta_{\text {pair }}\right|$. In the present setup, all the pair is created if typical comoving distance $x$ is on the order of the time $\eta_{\text {pair. }}$. See Figure 4 , where we have explicitly shown the particle creation process for mass profile A for two cases- $m \approx H$ and $m>>H$.

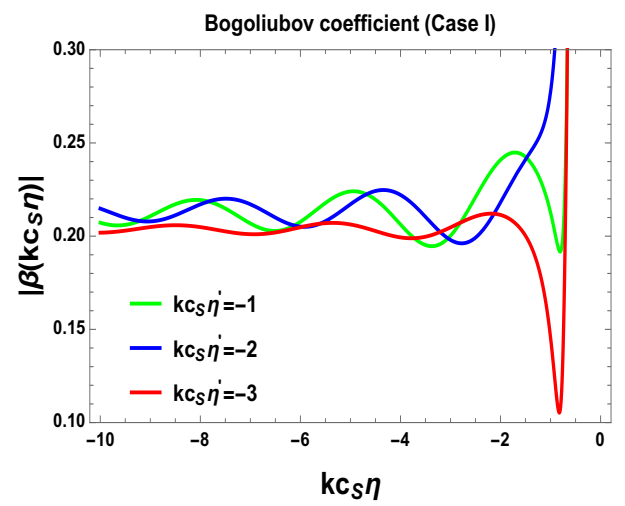

(a)

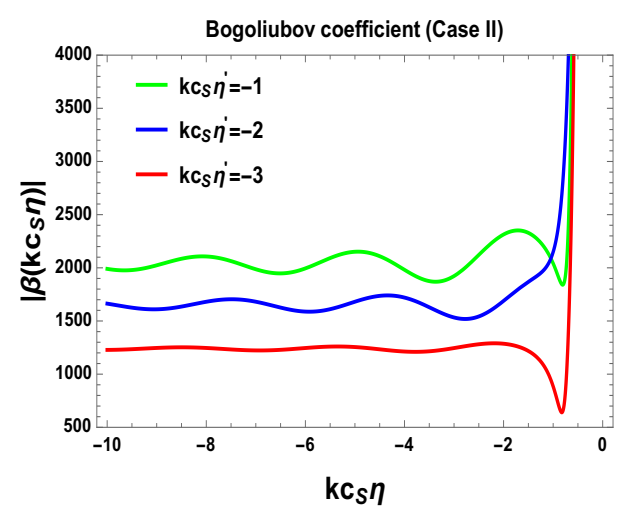

(c)

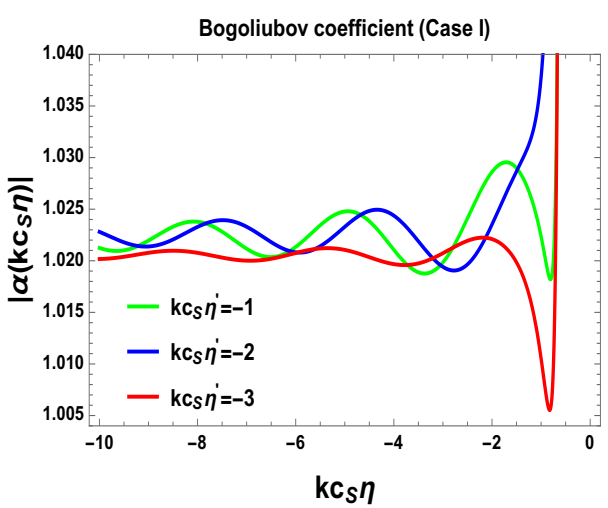

(b)

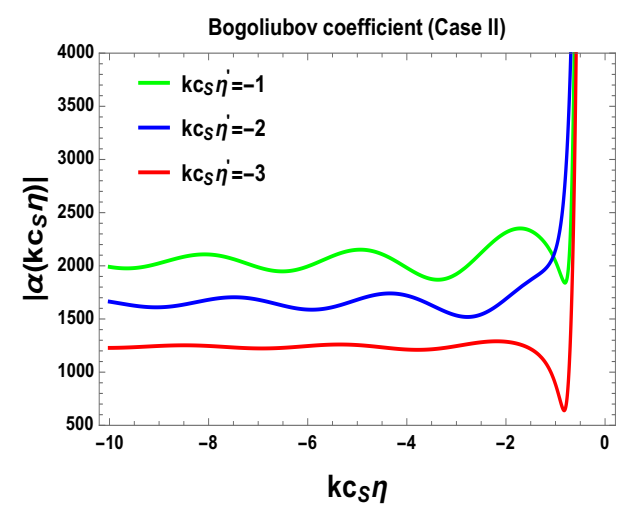

(d)

Figure 4. Particle creation for mass profile A for two cases: $m \approx H$ and $m>>H$. (a) For $m \approx H$; (b) For $m \approx H$; (c) For $m>>H$; (d) For $m>>H$. Here pictures are taken from [3]. 


\section{Important Note and Results in Cosmological Perturbation Theory}

Effect of the massive particles on the scalar curvature fluctuations can be described if we take the second-order action derived as [3]:

$$
S=\frac{1}{2} \int d \eta d^{3} x \frac{2 \epsilon M_{p}^{2}}{c_{S}^{2} H^{2}}\left[\frac{\left(\partial_{\eta} \zeta\right)^{2}-c_{S}^{2}\left(\partial_{i} \zeta\right)^{2}}{\eta^{2}}-\frac{m_{i n f}^{2}}{H^{2} \eta^{2}}\right]-\int \frac{d \eta}{c_{S} H} m(\eta) \partial_{\eta} \zeta(\eta, \mathbf{x}=0)
$$

Here, the curvature perturbation $\zeta$ is related to scalar field fluctuation as $\zeta=-H \delta \rho / \rho$, or more precisely in Fourier space, $\zeta_{\mathbf{k}}=-H \phi_{\mathbf{k}} / \dot{\bar{\phi}}_{0} M_{p}$. The last term contains the effect of massive particle, as the time-dependent mass parameter appears here. Additionally, it symbolizes the interaction term, where the inflaton field is interacting with the heavy fields. This is a complex model of inflation, as this model contains both inflaton and heavy field with a time-dependent coupling $m(\eta)$. However, in the simplest models of inflation, one can also neglect the mass contribution, because $m_{\text {inf }}<<H$ approximation is valid in those cases. We can compare our present setup with effective time varying mass parameter with the axions with time varying decay constant in the case of canonical interactions, which will be shown in the next section.

Using this action, one can further compute the one point function as:

$$
\begin{aligned}
\langle\zeta(\mathbf{x}, \eta=0)\rangle_{\left|k c_{s} \eta\right| \rightarrow-\infty} & \approx-\frac{2 H}{M_{p}^{2} \epsilon \pi}\left[\left|C_{2}\right|^{2} O_{1}-\left|C_{1}\right|^{2} O_{2}-i\left(C_{1}^{*} C_{2} e^{i \pi\left(\Lambda+\frac{1}{2}\right)}+C_{1} C_{2}^{*} e^{-i \pi\left(\Lambda+\frac{1}{2}\right)}\right) O_{3}\right], \\
\langle\zeta(\mathbf{x}, \eta=\xi \rightarrow 0)\rangle_{\left|k c_{S} \eta\right| \rightarrow 0} & =\frac{H \sqrt{\zeta} C_{S}}{2 M_{p}^{2} \epsilon \pi^{2}}\left(C_{1}^{*} C_{2}+C_{1} C_{2}^{*}-\left|C_{1}\right|^{2}-\left|C_{2}\right|^{2}\right) O_{4}^{\xi \theta} \\
\langle\zeta(\mathbf{x}, \eta=\xi \rightarrow 0)\rangle_{\left|k c_{S} \eta\right| \approx 1} & =\frac{H \sqrt{\xi} C_{S}}{2 M_{p}^{2} \epsilon \pi^{2}}\left(C_{1}^{*} C_{2}+C_{1} C_{2}^{*}-\left|C_{1}\right|^{2}-\left|C_{2}\right|^{2}\right) O_{5}^{\xi \Theta},
\end{aligned}
$$

where $\mathrm{O}_{1}, \mathrm{O}_{2}, \mathrm{O}_{3}, \mathrm{O}_{4}^{\xi \theta}, \mathrm{O}_{5}^{\xi \theta}$ are given by:

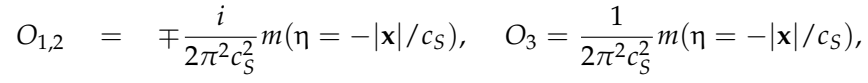

$$
\begin{aligned}
& O_{4}^{\tilde{\xi} \theta}=\frac{1}{2 \pi^{2}} \int_{0}^{\infty} d k k^{2} e^{i k x} \int_{-\infty}^{\tilde{\zeta}} d \eta \frac{c_{S}\left(\Lambda-\frac{1}{2}\right) m(\eta)}{a(\eta) \epsilon \sqrt{-\eta}}\left[\left(-\frac{k c_{S} \eta}{2}\right)^{-\Lambda}\left(-\frac{k c_{S} \tilde{\zeta}}{2}\right)^{-\Lambda}+\left(\frac{k c_{S} \eta}{2}\right)^{-\Lambda}\left(\frac{k c_{S} \tilde{\zeta}}{2}\right)^{-\Lambda}\right], \\
& O_{5}^{\tilde{\xi} \Theta}=\frac{1}{2 \pi^{2}} \int_{0}^{\infty} d k e^{i k x} k^{2-\Lambda} \int_{-\infty}^{\tilde{\xi}} d \eta \frac{c_{S}\left(\Lambda-\frac{1}{2}\right)\left(\frac{1}{2}\right)^{-\Lambda} m(\eta)}{a(\eta) \epsilon \sqrt{-\eta}}\left[\left(-\frac{c_{S} \xi}{2}\right)^{-\Lambda}+(-1)^{-\Lambda}\left(\frac{c_{S} \xi}{2}\right)^{-\Lambda}\right] .
\end{aligned}
$$

Here, $\xi$ is the cut-off in conformal time coordinates and acts as regulator in the theory and the parameter $\Lambda=\sqrt{v^{2}-m^{2} / H^{2}}$. Here it is important to mention that due to the presence of time-dependent mass parameter $\langle\zeta(\mathbf{x}, \eta=0)\rangle$ is non zero in all cases, and precisely shows the signature of Bell's inequality violation in primordial cosmology.

Similarly, in the position space, the two point function is given by:

$$
\begin{aligned}
\langle\zeta(\mathbf{x}, \eta) \zeta(\mathbf{y}, \eta)\rangle_{\left|k c_{S} \eta\right| \rightarrow-\infty} & \approx \frac{1}{4 \pi^{4}} \frac{H^{2}}{2 \epsilon M_{p}^{2}} \frac{\eta^{2} \tilde{c}_{S}^{2}}{c_{S}}\left[\left(\left|C_{2}\right|^{2}+\left|C_{1}\right|^{2}\right) J_{1}+\left(C_{1}^{*} C_{2} e^{i \pi\left(\Lambda+\frac{1}{2}\right)} J_{2}+C_{1} C_{2}^{*} e^{-i \pi\left(\Lambda+\frac{1}{2}\right)} J_{3}\right)\right], \\
\langle\zeta(\mathbf{x}, \eta) \zeta(\mathbf{y}, \eta)\rangle_{\left|k c_{S} \eta\right| \rightarrow 0} & \approx \frac{H^{2}}{2 \epsilon M_{p}^{2}} \frac{\left(-\eta c_{S}\right)^{3-2 \Lambda}}{2^{2(2-\Lambda)} \pi^{4}} \frac{\tilde{c}_{S}^{2}}{c_{S}^{3}}\left|\frac{\Gamma(\Lambda)}{\Gamma\left(\frac{3}{2}\right)}\right|^{2}\left[\left(\left|C_{2}\right|^{2}+\left|C_{1}\right|^{2}\right)-\left(C_{1}^{*} C_{2}+C_{1} C_{2}^{*}\right)\right] K_{I}, \\
\langle\zeta(\mathbf{x}, \eta=0) \zeta(\mathbf{y}, \eta=0)\rangle_{\left|k c_{S} \eta\right| \sim 1} & \approx \frac{H^{2}}{2 \epsilon M_{p}^{2}} \frac{1}{2^{2(2-\Lambda)} \pi^{4}} \frac{\tilde{c}_{S}^{2}}{c_{S}^{3}}\left|\frac{\Gamma(\Lambda)}{\Gamma\left(\frac{3}{2}\right)}\right|^{2}\left[\left(\left|C_{2}\right|^{2}+\left|C_{1}\right|^{2}\right)-\left(C_{1}^{*} C_{2}+C_{1} C_{2}^{*}\right)\right] Z_{I},
\end{aligned}
$$

where $J_{1}, J_{2}, J_{3}, K_{I}$, and $Z_{I}$ are defined as:

$$
J_{1}=-\frac{4 \pi}{|\mathbf{x}-\mathbf{y}|^{2}}, \quad J_{2,3}=-\frac{4 \pi}{\left(|\mathbf{x}-\mathbf{y}| \pm 2 c_{S} \eta\right)^{2}}, \quad K_{I}=4 \pi\left(\frac{i}{|\mathbf{x}-\mathbf{y}|}\right)^{3-2 \Lambda} \Gamma(3-2 \Lambda), Z_{I}=4 \pi\left\{\ln \left(\frac{L_{I R}}{|\mathbf{x}-\mathbf{y}|}\right)-\gamma_{E}\right\} .
$$


Here, $1 / L_{I R}$ is the infrared cut-off in the momentum scale which is introduced to regularize the momentum integral.

\section{Analogy with Axion Fluctuations from String Theory}

Let us consider the canonically normalized string theory originated axion action, which is represented as:

$$
S_{\text {axion }}=\int d \eta d^{3} x\left[\frac{f_{a}^{2}(\eta)}{2 H^{2}} \frac{\left[\left(\partial_{\eta} a\right)^{2}-\left(\partial_{i} a\right)^{2}\right]}{\eta^{2}}-\frac{U(a)}{H^{4} \eta^{4}}\right],
$$

where the dimensionless axion field $a=\phi / f_{a}$ with time-dependent decay constant $f_{a}$ and the corresponding potential can be given by:

$$
U(a)=\mu^{3} a f_{a}+\Lambda_{C}^{4} \cos a=\mu^{3} f_{a}[a+b \cos a],
$$

where the new parameter $b$ can be represented as $b=\frac{\Lambda_{C}^{4}}{\mu^{3} f_{a}}$. See Figure 5 , where we have shown the various parts of axion potential. Here in Equation (21), the linear part of the axion potential has been derived in the context of string theory in Reference [3], whereas the cosine part of the axion potential has its origin in non-perturbative aspects in string theory [3]. Here, only mass contribution for axion field will contribute to the fluctuations, whereas other part can be considered as back-reaction effect, which we can neglect since it is too small to be considered further. Thus, one can represent the axion potential as $U(a) \approx \frac{1}{2} m_{\text {axion }}^{2}\left(a-a_{0}\right)^{2}$. Additionally, it is important to mention that the scale $\Lambda_{C}$ is given by $\Lambda_{C}=\sqrt{m_{S U S Y} M_{p}} e^{-c S_{\text {inst }}}$, where $S_{\text {inst }}$ is the action of the instanton giving rise to effective potential, $c$ is constant factor on the order of unity, $m_{S U S Y}$ is the supersymmetry breaking mass scale, and $M_{p}$ represents reduced Planck mass, which is defined as $M_{p}=L^{3} / \sqrt{\alpha^{\prime}} g_{s}$. In this context, we assume that the axion decay constant $f_{a}$ is dependent on inflaton field, and initially this axion decay constant becomes large compared to the Hubble scale during inflation. During some time interval, it becomes smaller compared to the Hubble scale, a few e-foldings were created after the massive new particles, and then it becomes large again. Due to the increase in axion decay constant $f_{a}$, one can suppress quantum fluctuations effect at a shorter distance scale. Hence, we get an admissible value for the magnitude of the dimensionless axion field $a$ from axion at each created particle's location. As $f_{a}$ plays the same role as the mass parameter $m$, all of the earlier computations for four cases of the choice of mass parameters are valid here. Here the equation for the conformal time scale dependent axion decay constant is $f_{a}=\sqrt{100-\frac{80}{1+\left(\ln \frac{\eta}{\eta}\right)^{2}}} H$. In Table 1, we have shown the analogy between stringy axion and the new massive particle.

Table 1. The table below shows the analogy between the new particle and axion in string theory.

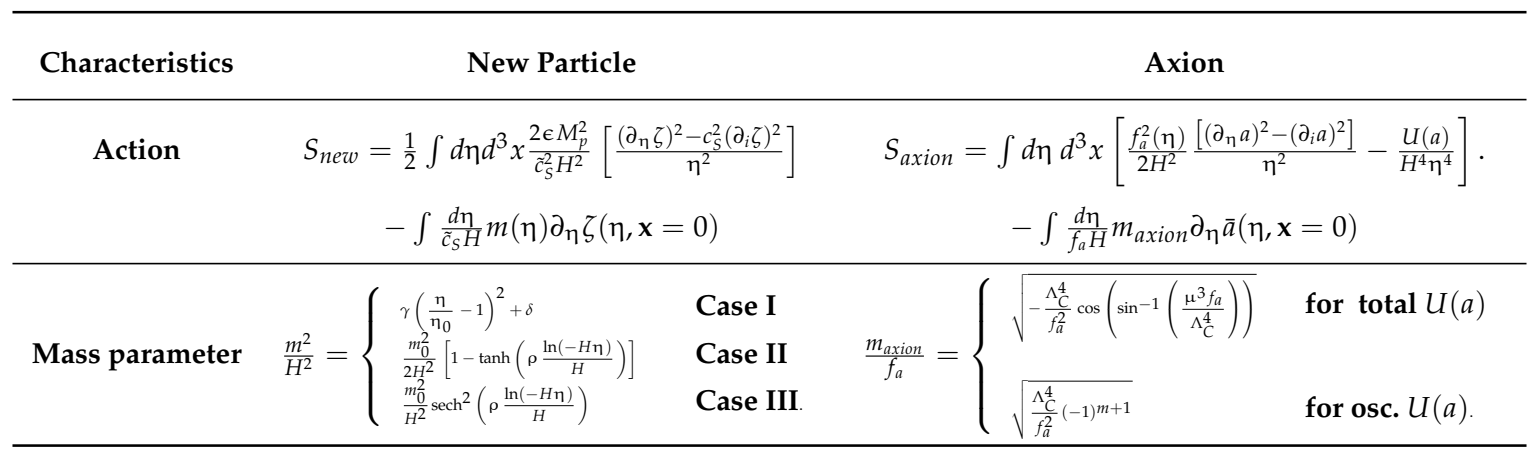


Table 1. Cont.

\begin{tabular}{|c|c|c|c|}
\hline Characteristics & New Particle & & Axion \\
\hline Rescaled mode & $h_{\mathbf{k}}=-\frac{\sqrt{2 \epsilon}}{H \tilde{H} \tilde{c} s} M_{p} \zeta$ & & $\vartheta_{\mathbf{k}}=\frac{f_{a}^{2}}{H^{2} \eta^{2} M_{p}^{2}} \bar{a}_{\mathbf{k}}$ \\
\hline Scalar mode & $h_{\mathbf{k}}^{\prime \prime}+\left(c_{S}^{2} k^{2}+\frac{\left(\frac{m^{2}}{H^{2}}-\delta\right)}{\eta^{2}}\right)$ & $h_{\mathbf{k}}=0$ & $\partial_{\eta}^{2} \vartheta_{\mathbf{k}}+\left(k^{2}-\frac{\partial_{\eta}^{2}\left(\frac{f_{a}^{2}}{H^{2} \eta^{2}}\right)}{\left(\frac{f_{a}^{2}}{H^{2} \eta^{2}}\right)}+\frac{m_{a x i o n}^{2}}{f_{a}^{2} H^{2} \eta^{2}}\right) \vartheta_{\mathbf{k}}=0$ \\
\hline Equation & where $\delta=\frac{z^{\prime \prime}}{z}=\left\{\begin{array}{l}2 \\
\left(v^{2}-\frac{1}{4}\right)\end{array}\right.$ & $\begin{array}{l}\text { for } d S \\
\text { for } q d S \text {. }\end{array}$ & where $\frac{\partial_{\eta}^{2}\left(\frac{f_{a}^{2}}{H^{2} \eta^{2}}\right)}{\left(\frac{f_{a}^{2}}{H^{2} \eta^{2}}\right)} \approx \begin{cases}\frac{6}{\eta^{2}} & \text { for } \eta \sim \eta_{c} \\
\frac{6}{\eta^{2}} & \text { for early and late } \eta \\
\frac{6+\Delta_{c}}{\eta^{2}} & \text { for } \eta<\eta_{c} .\end{cases}$ \\
\hline
\end{tabular}

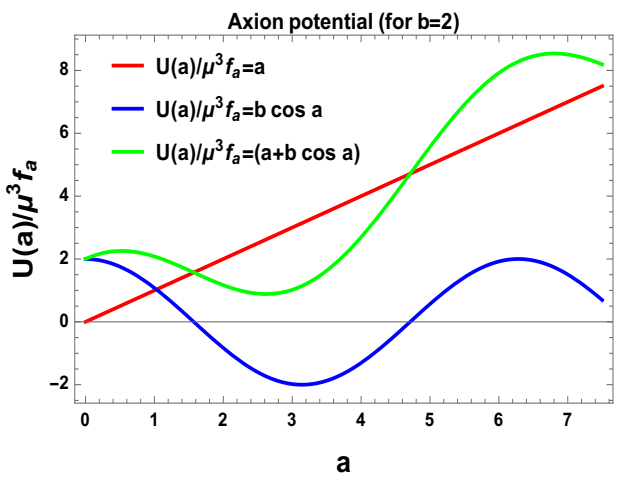

(a)

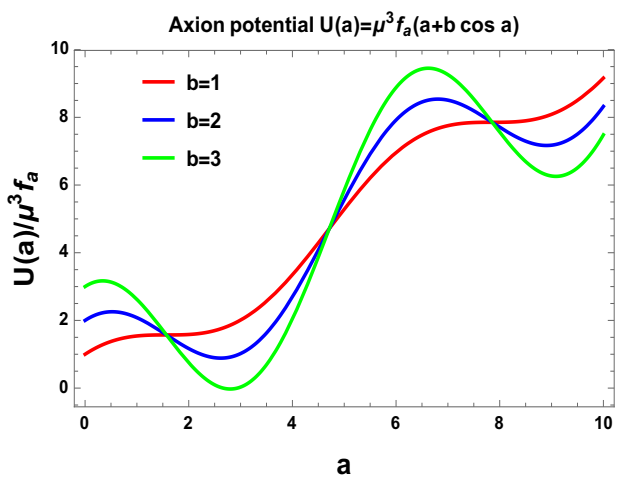

(b)

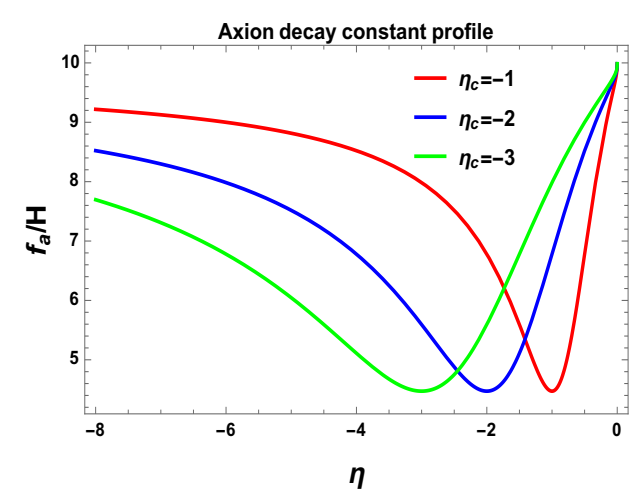

(c)

Figure 5. Behaviour of the axion effective potential and time-dependent decay constant. (a) Various parts of potential; (b) Total potential; (c) Axion decay constant profile. Here pictures are taken from [3].

\section{Role of Isospin Breaking Interaction and Detection}

We know that to construct a Bell violating experiment in the context of cosmology, isospin plays a very significant role. Therefore, let us consider the following effective Lagrangian for the inflaton and heavy scalar field interaction, which is given by:

$$
\begin{aligned}
S=\int d^{4} x \sqrt{-g}\left[\frac{1}{2} g^{\mu v}\left(\partial_{\mu} \mathcal{H}_{1}\right)^{*}\left(\partial_{\nu} \mathcal{H}_{1}\right)+\frac{1}{2} g^{\mu v}\left(\partial_{\mu} \mathcal{H}_{2}\right)^{*}\left(\partial_{\nu} \mathcal{H}_{2}\right)+\frac{1}{2} g^{\mu v}\left(\partial_{\mu} \phi\right)\left(\partial_{\nu} \phi\right)\right. \\
\left.-\left[\left|\mathcal{H}_{1}\right|^{2}+\left|\mathcal{H}_{2}\right|^{2}\right] \mathcal{M}_{\text {even }}^{2}(\phi)-\left[\exp (-i m \theta) \mathcal{H}_{1}^{*} \mathcal{H}_{2}+\exp (i m \theta) \mathcal{H}_{2}^{*} H_{1}\right] \mathcal{M}_{\mathbf{o d d}}^{2}(\phi)+\cdots\right] .
\end{aligned}
$$


Here $\phi$ is inflaton field, and the heavy field has an isospin $S U(2)$ doublet which is given by, $\mathcal{H}=\left(\mathcal{H}_{1}, \mathcal{H}_{2}\right)$. Here, the mass-terms are defined as $\mathcal{M}_{\text {even }}^{2}(\phi)=\sum_{n=0,2,4}^{\infty} \mathcal{M}_{n}^{2}(\phi), \mathcal{M}_{\text {odd }}^{2}(\phi)=$ $\sum_{n=1,3,5}^{\infty} \mathcal{M}_{n}^{2}(\phi)$. Using this, one can construct a physical mass eigenbasis from the eigenvalues: $\lambda_{ \pm}(\phi)=\sqrt{\mathcal{M}_{\text {even }}^{2}(\phi) \pm \mathcal{M}_{\text {odd }}^{2}(\phi)}$. To show the time-dependent behaviour of the mass parameters which are constructed out of the even and odd contributions in the interaction picture, here we chose I. $\lambda_{ \pm}(\eta) / H=\sqrt{\gamma_{ \pm}\left(\frac{\eta}{\eta_{0}}-1\right)^{2}+\delta_{ \pm}}$, II. $\lambda_{ \pm}(\eta) / H=\frac{m_{0 \pm}}{\sqrt{2} H} \sqrt{\left[1-\tanh \left(\rho \frac{\ln (-H \eta)}{H}\right)\right]}$, III. $\lambda_{ \pm}(\eta) / H=$ $\frac{m_{0 \pm}}{H} \operatorname{sech}\left(\rho \frac{\ln (-H \eta)}{H}\right)$. To simplify the calculations, we introduce new parameters as: $\gamma_{ \pm}=\gamma_{\text {even }} \pm$ $\gamma_{\text {odd }}, \delta_{ \pm}=\delta_{\text {even }} \pm \delta_{\text {odd }}, m_{0 \pm}=\sqrt{m_{\text {even }}^{2} \pm m_{\text {even }}^{2}}$. See Figure 6, where we have shown the conformal time dependent behaviour of three toy models of heavy particle mass profile for two eigenstates. To make the eigenbasis stable, eigenvalues of the mass matrix are always positive definite. At late time scales $\mathcal{M}_{\text {even }}^{2}(\phi) \sim \mathcal{M}_{\text {odd }}^{2}(\phi)$. Hence, the eigenvalue of the mass-matrix increases. Another important requirement is that eigenvalues of the mass matrix should be on the order of $M_{p}$. The eigenvalue of the mass matrix is $\lambda(\phi) \sim \mathcal{M}_{\text {even }}(\phi)$ when we know that all the isospin breaking interactions are absent from the effective Lagrangian. This is also one criterion of significant importance that can be observed with $S U(2)$ isospin singlet state during the time of heavy mass particle creation. Here the angular parameter $\theta$ and its functional dependence on the background plays an extremely important role to set up the Cosmological Bell violating setup. To make the case easy, one can assume that $\theta$ is a constant. Here, if the particle mass eigenvalue is $\lambda_{ \pm}(\phi)$, then the antiparticle mass eigenvalues are given by $\lambda_{ \pm}(\phi)$. The sign of the eigenvalue of the antiparticle mass eigenstate may flip if the angular parameter $\theta$ is not a constant quantity but background dependent. After the period of inflation, we can set the axion potential to be zero to avoid the problem of domain wall formation. We have not yet observed any signatures of these heavy fields or the axion; therefore, we can consider such heavy fields or the axions corresponding to a component of dark matter, and we can treat its corresponding density fluctuations as isocurvature fluctuations.

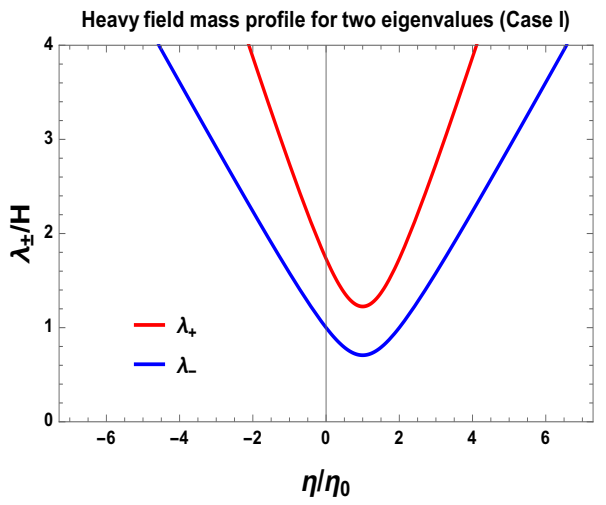

(a)

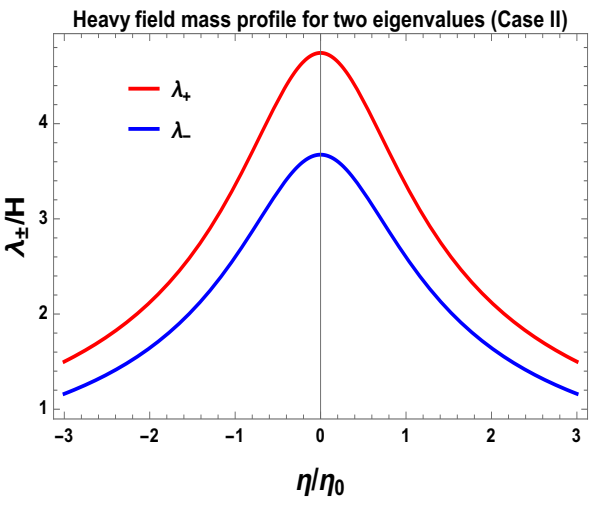

(b)

Figure 6. Cont. 


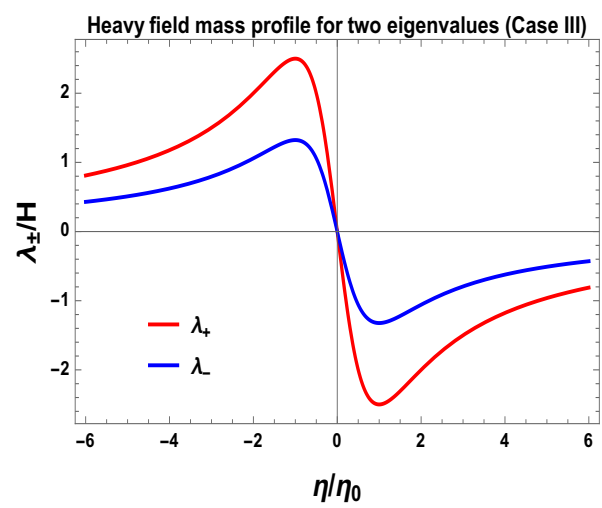

(c)

Figure 6. Conformal time scale dependent behaviour of heavy particle mass profile for two eigenstates. (a) Here we set $\gamma_{\text {even }}=1=\delta_{\text {even }}, \gamma_{\text {odd }}=0.5=\delta_{\text {odd }} ;(\mathbf{b})$ Here we set $m_{\text {even }}=3, m_{\text {odd }}=1.5$ and $\rho / H=1 ;$ (c) Here we set $m_{\text {even }}=2, m_{\text {odd }}=1.5$ and $\rho / H=1$. Here pictures are taken from [3].

\section{Role of Spin for New Particles}

Let us generalize the idea where we consider the situation for a dynamical massive field with arbitrary spin $\mathcal{S}$. Therefore, in this case, the classical time dependence of the high spin modes give rise to time-dependent mass $m_{\mathcal{S}}(\eta)$ for the spin field. Hence, for the massive field with arbitrary spin $\mathcal{S}$, the equation of motion is given by:

$$
h_{k}^{\prime \prime}+\left\{c_{S}^{2} k^{2}+\left(\frac{m_{\mathcal{S}}^{2}}{H^{2}}-\left[v_{\mathcal{S}}^{2}-\frac{1}{4}\right]\right) \frac{1}{\eta^{2}}\right\} h_{k}=0,
$$

where in quasi de-Sitter case, $v_{\mathcal{S}}=\left(\mathcal{S}-\frac{1}{2}\right)+\epsilon+\frac{\eta}{2}+\frac{s}{2}$. Now, following the same procedure mentioned earlier, we finally give the estimate of the mass parameter as $\left|\frac{m_{\mathcal{S}}}{H}\right|=\left|\sqrt{v_{\mathcal{S}}^{2}-\frac{\left(4-n_{\zeta}\right)^{2}}{4}}\right| \geq$ $\sqrt{v_{\mathcal{S}}^{2}-\frac{1}{4}}$, where $n_{\zeta}$ is scalar spectral tilt, which is constrained from cosmological observation (Planck 2015 data).

\section{Conclusions}

To summarize, in the present article we have addressed following important points:

- We provide a toy model for Bell's inequality violation in cosmology.

- The model consists of inflaton and additional massive field with time-dependent behaviour.

- For each model, massive particle creation in "isospin" singlet state plays a crucial role.

- The prescribed methodology is consistent with axion fluctuations appearing in the context of String Theory.

- The signature of the Bell violation is visualized from non-zero one-point function of curvature fluctuation. Additionally, we provide the result of a two-point function which has direct observational consequence.

- Finally, we also provide the mass bound on the new particle in terms of the arbitrary spin $\mathcal{S}$.

Acknowledgments: S.C. would like to thank Department of Theoretical Physics, Tata Institute of Fundamental Research, Mumbai for providing me Visiting (Post-Doctoral) Research Fellowship. S.C. would like to thank Gautam Mandal and Guruprasad Kar for useful discussions and suggestions. S.C. take this opportunity to thank sincerely to Sandip P. Trivedi and Shiraz Minwalla for their constant support and inspiration. S.C. also thanks the organizers of VARCOSMOFUN'16 where this work was presented. Last but not the least, we would all like to acknowledge our debt to the people of India for their generous and steady support for research in natural sciences, especially for string theory and cosmology.

Author Contributions: The authors contribute equally to this paper. 
Conflicts of Interest: The authors declare no conflict of interest.

\section{References}

1. Bell, J.S. On the Einstein-Podolsky-Rosen paradox. Physics 1964, 1, 195-200.

2. Maldacena, J. A model with cosmological Bell inequalities. Fortschr. Phys. 2016, 64, 10-23.

3. Choudhury, S.; Panda, S.; Singh, R. Bell violation in the Sky. Eur. Phys. J. C 2016, 77 , 60.

4. Maldacena, J. Talk. Available online: https://sites.google.com/site/andyfest2015/program (accessed on 9 November 2016).

5. Reid, M.D.; Walls, D.F. Violations of classical inequalities in quantum optics. Phys. Rev. A 1986, $34,1260$.

6. Holman, R.; Mersini-Houghton, L.; Takahashi, T. Cosmological avatars of the landscape. II. CMB and LSS signatures. Phys. Rev. D 2008, 77, 063511.

7. Kanno, S. Cosmological implications of quantum entanglement in the multiverse. Phys. Lett. B 2015, 751, 316-320.

8. Robles-Pérez, S.; González-Díaz, P.F. Quantum entanglement in the multiverse. J. Exp. Theor. Phys. 2014, 118, 34-53.

(C) 2017 by the authors; licensee MDPI, Basel, Switzerland. This article is an open access article distributed under the terms and conditions of the Creative Commons Attribution (CC BY) license (http:/ / creativecommons.org/licenses/by/4.0/). 